\title{
Segurança, Eficácia e Protocolo de Dose de Metoprolol para Redução de Frequência Cardíaca em Pacientes Pediátricos Externos que Passaram por Angiografia Cardíaca por TC
}

Safety, Efficacy, and Dose Protocol of Metoprolol for Heart Rate Reduction in Pediatric Outpatients Undergoing Cardiac CT Angiography

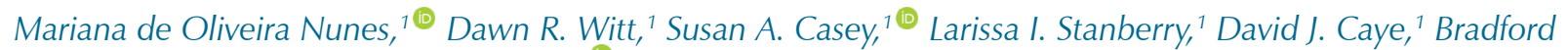
J.Chu, ${ }^{2}$ B. Jana Lindberg, ${ }^{1}$ John R. Lesser, ${ }^{10}$ B. Kelly Han ${ }^{1,2}$

Minneapolis Heart Institute and Foundation, ${ }^{1}$ Minnesota - EUA

Children's Minnesota, Minneapolis, ${ }^{2}$ Minnesota - EUA

\section{Resumo}

Fundamento: Qualidade de imagem e dose de radiação são otimizadas com uma frequência cardíaca (FC) lenta e estável na realização de imagens de artérias coronárias durante a angiografia cardíaca por tomografia computadorizada (CCTA, do inglês cardiac computed tomography angiography) A segurança, a eficácia e o protocolo para a redução da FC com medicamento betabloqueador ainda não foi bem descrita em uma população de pacientes pediátricos.

Objetivo: Oferecer um protocolo de dose de metoprolol eficiente a ser usado em pacientes pediátricos externos durante a CCTA.

Métodos: Realizamos uma revisão retrospectiva de todos os pacientes pediátricos externos que receberam o metoprolol durante a CCTA. As características demográficas e clínicas foram resumidas e a redução média em FC foi estimada utilizando-se um modelo de regressão linear multivariada. As imagens foram avaliadas em uma escala de 1 a 4 (1 = ideal).

Resultados: Um total de 78 pacientes externos passaram a uma CCTA com o uso de metoprolol. A média de idade foi de 13 anos, a média de peso foi de $46 \mathrm{~kg}$, e 36 pacientes (46\%) eram do sexo masculino. As doses médias de metoprolol foram 1,5 (IQR 1,1; 1,8) $\mathrm{mg} / \mathrm{kg}$, e 0,4 (IQR 0,2; 0,7) $\mathrm{mg} / \mathrm{kg}$ para administrações orais e intravenosas, respectivamente. O produto dose-comprimento por exame foi de 57 (IQR 30, 119) $\mathrm{mGy} * \mathrm{~cm}$. A redução média da FC foi 19 (IQR 12, 26) batimentos por minuto, ou $23 \%$. Não foram relatadas complicações ou eventos adversos.

Conclusão: $\mathrm{O}$ uso de metoprolol num cenário de pacientes pediátricos externos para redução da FC antes de uma CCTA é seguro e eficiente. Pode-se reproduzir um protocolo de dose de metoprolol quando for necessário atingir uma FC mais lenta, garantindo tempos de aquisição mais rápidos, imagens mais claras e redução na exposição à radiação nessa população. (Arq Bras Cardiol. 2021; 116(1):100-105)

Palavras-chave: Cardiopatias Congênitas; Frequência Cardíaca; Metoprolol; Antagonistas Adrenérgicos;Tomografia Computadorizada; Vasos Coronários.

\begin{abstract}
Background: Image quality and radiation dose are optimized with a slow, steady heart rate (HR) when imaging the coronary arteries during cardiac computed tomography angiography (CCTA). The safety, efficacy, and protocol for HR reduction with beta blocker medication is not well described in a pediatric patient population.
\end{abstract}

Objective: Provide a safe and efficient metoprolol dose protocol to be used in pediatric outpatients undergoing CCTA.

Methods: We conducted a retrospective review of all pediatric outpatients who received metoprolol during CCTA. Demographic and clinical characteristics were summarized and the average reduction in HR was estimated using a multivariate linear regression model. Images were evaluated on a 1-4 scale (1 = optimal).

Correspondência: Mariana de Oliveira Nunes •

Minneapolis Heart Institute Foundation - 920 East 28th st suite 610 Minneapolis, Minnesota 55407-1195 - EUA

E-mail: nunes.mo@gmail.com

Artigo recebido em 17/12/2019, revisado em 12/06/2020, aceito em 05/08/2020

DOI: https://doi.org/10.36660/abc.20190892 
Results: Seventy-eight pediatric outpatients underwent a CCTA scan with the use of metoprolol. The median age was 13 years, median weight of $46 \mathrm{~kg}$, and $36(46 \%)$ were male. The median doses of metoprolol were $1.5(I Q R ~ 1.1,1.8) \mathrm{mg} / \mathrm{kg}$ and $0.4(I Q R ~ 0.2,0.7) \mathrm{mg} / \mathrm{kg}$ for oral and intravenous administrations, respectively. Procedural dose-length product was $57(I Q R 30,119) \mathrm{mGy}{ }^{*} \mathrm{~cm}$. The average reduction in HR was 19 $(I Q R$ 12, 26) beats per minute, or $23 \%$. No complications or adverse events were reported.

Conclusion: Use of metoprolol in a pediatric outpatient setting for HR reduction prior to CCTA is safe and effective. A metoprolol dose protocol can be reproduced when a slower HR is needed, ensuring faster acquisition times, clear images, and associated reduction in radiation exposure in this population. (Arq Bras Cardiol. 2021; 116(1):100-105)

Keywords: blHeart Defects, Congenital; Heart Rate; Metoprolol; Adrenergic, Antagonists; Computed, Tomography; Coronary Vessels.

Full texts in English - http://www.arquivosonline.com.br

\section{Introdução}

A angiografia cardíaca por tomografia computadorizada (CCTA) é o padrão de imagem para avalição não invasiva das artérias coronárias em pacientes de todas as idades. ${ }^{1,2}$ Para otimizar a qualidade da imagem e a dose de radiação, prefere-se ter uma FC mais baixa e estável. ${ }^{3,4}$ A redução da FC pode ser alcançada pelo uso de medicamento betabloqueador. Exames de imagem de artérias coronárias em crianças apresentam desafios específicos, devido ao tamanho dos vasos e FC mais alta em repouso. A principal modalidade diagnóstica para imagem coronária em pacientes com doença cardíaca congênita (DCC) historicamente tem sido a cateterização cardíaca, que exige anestesia, acesso vascular central, administração de contraste, e exposição significativa à radiação. A imagem cardíaca por ressonância magnética é útil para a imagem coronária, mas tem valor limitado em pacientes mais jovens. ${ }^{5}$ A CCTA demonstrou ser uma forma de diagnóstico em bebês e crianças de todas as idades, utilizando tomógrafos de última geração com resolução espaço-temporal adequada. ${ }^{6-8}$

Técnicas de otimização de dose de radiação diminuíram significativamente a exposição em comparação com tecnologias de tomografia anteriores. A imagem coronária pode ser adquirida em um único batimento ou em alguns batimentos cardíacos durante uma apnéia em pacientes de todas as idades. ${ }^{9}$ A FC mais baixa e estável permite o uso de uma janela estreita de aquisição de exposição à radiação, durante a sístole ou a diástole, dependendo da FC. A segurança e a eficácia da redução da FC com medicamento betabloqueador já está bem descrita para imagem coronária na população adulta, ${ }^{10-12}$ mas é rara para pacientes pediátricos. ${ }^{6,13} \mathrm{O}$ objetivo desse estudo retrospectivo foi avaliar a segurança e a eficácia, e definir o protocolo de dosagem de metoprolol para redução da FC em uma população de pacientes pediátricos externos que passaram por CCTA.

\section{Métodos}

\section{Pacientes}

Foram incluídos no estudo pacientes entre 6 e 18 anos de idade que compareceram como pacientes externos e receberam o metoprolol antes da CCTA, no período de $1 \stackrel{0}{ }$ de janeiro de 2007 a 31 de dezembro de 2016. Pacientes que fizeram a TC por indicação que não fosse uma DCC, que fizeram a CCTA sem o medicamento metoprolol, que foram encaminhados para a imagem coronária como pacientes internos, ou que eram pacientes externos, mas fizeram a tomografia anestesiados para garantir a cooperação com a suspensão da respiração foram excluídos do estudo. A FC de linha de base foi medida na apresentação do paciente externo no centro de imagens antes da administração do metoprolol e novamente durante a tomografia. A dose de metoprolol, a qualidade da imagem, e quaisquer eventos adversos foram documentados. O estudo foi aprovado pelo Comitê de Análise Institucional.

\section{Plataforma do Tomógrafo e Sequência de Varredura, e Preparação do Paciente}

A CCTA foi realizada utilizando-se um tomógrafo de fonte dupla de primeira, segunda ou terceira geração (Somatom Definition Flash, Siemens Healthcare, Forchheim, Alemanha) com tempo de rotação do tubo $=280 \mathrm{~ms}$, resolução temporal =66-83 ms, e colimação $=2 \times 128 \times 0,6 \mathrm{~mm}$. Uma CCTA sincronizada ao eletrocardiograma (ECG), prospectiva e com alto pitch $(3,4)$ foi realizada utilizando-se modulação de corrente de tubo online automatizada para uma FC lenta e estável $<55$ batimentos por minuto (bpm), com tomógrafo de fonte dupla de segunda ou terceira geração. Nos casos de FC mais alta ou irregularidade significativa de FC, apesar do betabloqueador, foi realizado uma aquisição retrospectiva sincronizada ao ECG (Mindose) ou uma aquisição sequencial foi feita com a janela de aquisição ajustada conforme a FC. Tipicamente, foi utilizada uma janela de aquisição mais larga que incluiu a sístole para FC acima de 60 bpm. Quando se suspeitou de lesões coronárias nos pacientes com sintomas de isquemia ou doença de Kawasaki, foi usado uma aquisição retrospectiva sincronizada ao ECG (Mindose) ou uma aquisição sequencial, independente da FC, para permitir a avaliação de mais de um único conjunto de dados. Para todos os pacientes, a voltagem do tubo foi ajustado a um valor mais baixo baseado no uso do software automatizado Care kV (Siemens, Forchheim) ou na avaliação clínica. Em 2011, a voltagem do tubo a $70 \mathrm{kV}$ foi disponibilizado com uma melhoria do tomógrafo. Os exames foram reconstruídos usando o algoritmo de reconstrução iterativa de segunda geração da Siemens, o Safire, na potência 3. Em 2014, uma terceira geração de algoritmos de reconstrução iterativa, o Admire, passou a ser usado, também na potência 3. A dose de contraste foi injetada na velocidade apropriada para a idade e o calibre intravenoso. O contraste foi injetado através de bomba injetora utilizando um cateter de calibre 20-24, com base no tamanho do paciente. 


\section{Avaliação da Qualidade da Imagem}

As imagens foram analisadas retrospectivamente por dois leitores especialistas (KH e BC), qualitativamente, conforme uma escala de quatro pontos: $1=$ totalmente aceitável com visualização ideal de todos os alvos anatômicos; $2=$ boa qualidade de imagem com visualização diagnóstica de todos os alvos anatômicos; $3=$ qualidade marginal da imagem, com visualização da maioria dos alvos anatômicos; e 4=má qualidade da imagem, não diagnóstica para a avaliação dos alvos anatômicos. Quaisquer discrepâncias na pontuação da qualidade da imagem foram revisadas novamente por $\mathrm{KH}$ e resolvidas. Os alvos anatômicos foram definidos como a capacidade de definição clara dos óstios coronários e a sua origem da grande artéria, definição clara do trajeto da coronária incluindo o relacionamento com as grandes artérias e o esterno, e a capacidade de identificar a anatomia da coronária distal para determinar a dominância coronária. Todas as tomografias com uma pontuação $>1$ foram consideradas abaixo do ideal. Para essas tomografias, foi determinado o motivo para classificação da imagem como subótima.

\section{Estimativa da Dose de Radiação}

O produto dose-comprimento do procedimento, em mGy*cm foi utilizada para estimar a dose de radiação. Um cilindro de $32 \mathrm{~cm}$ foi usado para estimativas de produto dosecomprimento em todos os pacientes, independentemente do tamanho. A dose de radiação é relatada como sendo o produto dose-comprimento do exame.

\section{Protocolo de Administração de Metoprolol}

Um protocolo de metoprolol padrão foi usado para todos os pacientes incluídos neste estudo. As crianças passaram por triagem para determinar as contraindicações do uso de betabloqueador, incluindo estenose aórtica grave, hipertensão pulmonar de moderada a grave, ou disfunção sistólica ventricular esquerda ou direita grave. Pacientes com histórico de qualquer uma dessas entidades clínicas não receberam o betabloqueador. Quando a FC basal era $<60$ bpm, o metoprolol não foi administrado. Quando a FC basal ficava entre 60 e $70 \mathrm{bpm}, 1 \mathrm{mg} / \mathrm{kg}$ de metoprolol, até a dose máxima de $100 \mathrm{mg}$, foi administrado. Quando a FC basal era $>70 \mathrm{bpm}, 2 \mathrm{mg} / \mathrm{kg}$ de metoprolol, até a dose máxima de $100 \mathrm{mg}$, foram administrados. Quando a FC permanecia acima de 70 bpm uma hora após a dose oral, 0,2 mg/kg de metoprolol intravenoso era administrada até a dose máxima de $1 \mathrm{mg} / \mathrm{kg}$, para pacientes $<20 \mathrm{~kg}$, ou dose máxima de $20 \mathrm{mg}$ de dose intravenosa total, eram administrados para pacientes com peso acima de $20 \mathrm{~kg}$. Se a FC no tomógrafo estiver $>70$ bpm quando a FC basal tiver sido aceitável, o metoprolol intravenoso só foi administrado de acordo com as diretrizes acima. ${ }^{14}$

\section{Métodos Estatísticos}

Os dados demográficos e clínicos do paciente foram resumidos usando contagens (\%) para variáveis categóricas, médias \pm desvio padrão para variáveis contínuas simetricamente distribuídas, e medias (faixas interquartis) para variáveis contínuas distorcidas. A mudança da FC após a administração do betabloqueador foi estimada utilizando-se um modelo de regressão linear multivariada, com a diferença em FC como variável de resposta, e idade, sexo, o produto dosecomprimento e a dose de metoprolol como covariáveis. As premissas do modelo foram definidas usando-se análise residual e o teste Shapiro-Wilk de normalidade. As estimativas do modelo, seus intervalos de confiança $(\mathrm{Cl})$ de $95 \%$, e os $\mathrm{p}$-valores foram relatados. A análise foi realizada usando o $\mathrm{R}$ 3.5.2 em ambiente R-Studio 1.1.463. ${ }^{14,15}$ Foi usado um nível de significância de 5\%.

\section{Resultados}

\section{Dados Demográficos dos Pacientes e Redução da Frequência Cardíaca}

Identificamos 78 pacientes pediátricos que passaram por uma CCTA com o uso de metoprolol antes da aquisição da imagem no contexto de pacientes externos em nossa instituição entre janeiro de 2007 e dezembro de 2016. Destes, cinquenta e nove $(75 \%)$ pacientes fizeram a tomografia para avaliar as coronárias, e 19 (25\%) a fizeram para avaliar outro tipo de DCC. Os dados demográficos dos pacientes, a FC e o mecanismo de administração do betabloqueador estão descritos na Tabela 1. A média de idade na tomografia foi 13,33 (IQR 10, 16) anos de idade, 36 pacientes (46\%) eram do sexo masculino, e a média de peso foi de 46 (IQR 31, 61) $\mathrm{kg}$. Um paciente recebeu nitroglicerina sem efeito adverso.

No geral, a FC de linha de base foi de 77 (IQR 66, 90) bpm. A maioria dos pacientes, $\mathrm{n}=51$, (65\%) recebeu apenas o metoprolol por via oral, e quatro pacientes (5\%) receberam apenas o metoprolol intravenoso. Os demais pacientes receberam uma combinação de metoprolol via oral e intravenoso, $n=23$ (29\%). Após a administração do metoprolol, houve uma redução de $23 \%$ na FC basal que corresponde a 19 bpm, IQR (12-26). A partir da análise multivariada, a redução estimada na FC foi 20 bpm, com 95\% IC $(17,24)$ (Apêndice 1).

\section{Administração de Metoprolol}

A dose de metoprolol depende do peso do paciente, conforme definido no Protocolo de administração de metoprolol, descrito anteriormente. Para os pacientes que pesavam $\leq 50 \mathrm{~kg}$, a dose média de metoprolol oral ou intravenoso foi de 1,6 mg/kg (IQR 1,3, 1,9) e 0,6 mg/ kg (IQR 0,3,0,8), respectivamente. Para os pacientes que pesavam acima de $50 \mathrm{~kg}$, a dose média de metoprolol oral ou intravenoso foi de 1,4 mg/kg (IQR 1,0, 1,6) e 0,3 mg/ kg (IQR 0,1,0,5), respectivamente. (Tabela 2) As doses e quantidades administradas na prática eram consistentes com as especificadas em nosso protocolo clínico. ${ }^{14}$

\section{Dose de Radiação e Detalhes da Imagem}

A Tabela 3 apresenta a dose de radiação da tomografia e detalhes da imagem. A média do produto dose-comprimento do exame foi de 57 (IQR 30, 119) mGy*cm. A pontuação média da qualidade da imagem foi de 1,2. Das 78 tomografias, 


\begin{tabular}{|c|c|c|c|c|}
\hline Variável & Todos & Apenas oral & Apenas IV & IV + Oral \\
\hline Paciente, $\mathrm{n}(\%)$ & $78(100)$ & $51(65)$ & $4(5)$ & $23(29)$ \\
\hline Idade na tomografia, anos & $13,0 \pm 3,3$ & $13,1 \pm 3,4$ & $10,2 \pm 4,6$ & $13,3 \pm 2,7$ \\
\hline Sexo masculino, $n(\%)$ & $36(46)$ & $22(43)$ & $1(25)$ & $13(57)$ \\
\hline Peso, kg, ${ }^{*}$ & $46(31,61)$ & $46(29,59)$ & $32(29,58)$ & $49(36,62)$ \\
\hline FC inicial, bpm, ${ }^{*}$ & $78 \pm 15$ & $74 \pm 11$ & $91 \pm 26$ & $87 \pm 16$ \\
\hline FC na tomografia, bpm, ${ }^{*}$ & $60 \pm 11$ & $56 \pm 9$ & $73 \pm 16$ & $66 \pm 11$ \\
\hline Redução de FC, bpm, ${ }^{*}$ & $19 \pm 10$ & $18 \pm 9$ & $18 \pm 10$ & $20 \pm 12$ \\
\hline Redução relativa da FC, \%, & $23(16,30)$ & $24(17,30)$ & $20(17,22)$ & $23(15,33)$ \\
\hline
\end{tabular}

N: número; IV: intravenosa; kg: quilograma; FC: frequência cardiaca; bpm: batimentos por minuto. * Variáveis contínua são relatadas como \pm desvios padrão médios ou como faixas médias e interquartis (IQR, $25^{\circ}, 75^{\circ}$ percentis) se distorcidas. Variáveis categóricas resumidas por contagens (\%).

Tabela 2 - Protocolo de Betabloqueadores - Dose e entrega por peso

\begin{tabular}{lccc}
\hline Variável & Todos & Peso $\leq 50 \mathrm{~kg}$ & Peso $>\mathbf{5 0} \mathrm{kg}$ \\
\hline Dose oral, $\mathrm{mg} / \mathrm{kg}\left(\mathrm{n}=74^{*}\right)$ & $1.5 \pm 0.5$ & $1.7 \pm 0.5$ & $1.3 \pm 0.4$ \\
\hline Dose IV, $\mathrm{mg} / \mathrm{kg}\left(\mathrm{n}=27^{*}\right)$ & $0.5 \pm 0.3$ & $0.6 \pm 0.3$ & $0.4 \pm 0.2$ \\
\hline Quantidade oral, $\mathrm{mg}$ & $50(50,100)$ & $50(50,75)$ & $100(62,100)$ \\
\hline Quantidade IV, $\mathrm{mg}$ & $20(15,28)$ & $20(13,23)$ & $20(18,35)$ \\
\hline
\end{tabular}

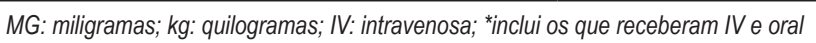

Variáveis contínua são relatadas como \pm desvios padrão médios ou como faixas médias e interquartis (IQR, $25^{\circ}, 75^{\circ}$ percentis) se distorcidas.

Tabela 3 - Dose de radiação da tomografia e detalhes da imagem

\begin{tabular}{lr}
\hline DLP, $m \mathrm{my}^{*} \mathrm{~cm}{ }^{*}$ & $57(30,119)$ \\
\hline Qualidade da imagem da tomografia* & \\
\hline $1, \mathrm{n}(\%)(\%)$ & $67(86)$ \\
\hline $2, \mathrm{n}(\%)(\%)$ & $10(13)$ \\
\hline $3, \mathrm{n}(\%)(\%)$ & $1(1)$ \\
\hline $4, \mathrm{n}(\%)(\%)$ & $0(0)$ \\
\hline Sequência de imagem & $26(33)$ \\
\hline ECG anatômico-prospectivo disparado com alta frequência (Flash), $\mathrm{n}(\%)$ & $25(32)$ \\
\hline ECG anatômico-prospectivo disparado (Prospectivo), $\mathrm{n}(\%)$ & $27(35)$ \\
\hline ECG retrospectivo-funcional (espiral), $\mathrm{n}(\%)$ & \\
\hline
\end{tabular}

DLP: produto dose-comprimento (do inglês dose-length product); $m G y^{*} \mathrm{~cm}$. " variáveis categóricas resumidas por contagens (\%); variáveis contínuas relatadas como médias e faixas de interquartil (IQR, $25^{\circ} 75^{\circ}$ percentis). Descrição da qualidade da imagem $1=$ totalmente aceitável com visualização ideal de todos os alvos anatômicos; 2=boa qualidade de imagem com visualização diagnóstica de todos os alvos anatômicos; 3=qualidade marginal da imagem, com visualização da maioria dos alvos anatômico; e 4=má qualidade da imagem, não diagnóstica para a avaliação de alvos anatômicos

11 (14\%) ficaram abaixo da qualidade ideal, com 10 casos pontuados como "2" devido ao contraste ruim e/ou ruído, e um caso foi classificado como "3", devido a movimento do paciente. A representação das sequências de imagem foi uniforme, com aproximadamente um terço dos pacientes incluídos em cada tipo de sequência. Não foram relatadas complicações durante os procedimentos de imagem por CCTA ou depois do procedimento até o momento da alta do ambiente de paciente externo. ${ }^{15}$

\section{Discussão}

Em pacientes adultos submetidos a CCTA, o uso de betabloqueador com controle da FC adequada demonstrou que melhora a qualidade da imagem. ${ }^{16}$ A pré-medicação por via oral demonstrou ser eficiente na população adulta, embora a variação da eficácia seja afetada pela dosagem. ${ }^{16}$ Já está bem documentado que os riscos de exposição repetida a anestesia e radiação ionizante devem ser evitadas para todos os pacientes de DCC. ${ }^{17-21}$ Portanto, uma FC mais lenta 
permite o uso de eletrocardiograma prospectivo de disparo, que demonstrou reduzir significativamente a dose para angiografia coronária. ${ }^{22}$ Em nossa experiência, o uso de metoprolol intravenoso uma hora após a dose oral não teve efeito adicional na redução da FC. Portanto, interrompemos a administração de metoprolol intravenoso após a dose oral em nossa população de pacientes pediátricos a partir de 2013. Deve-se notar que três pacientes receberam o metoprolol IV depois de 2013 devido a FC elevada durante a aquisição do topograma por sofrerem de ansiedade. A redução da FC nas populações pediátricas pode ser alcançada com segurança e eficiência com um protocolo de administração de metoprolol padronizado, para pacientes submetidos a avaliação de CCTA no contexto de pacientes externos. Com triagem cuidadosa para contraindicações, não encontramos complicações ou efeitos colaterais com o uso de betabloqueadores em pacientes pediátricos.

\section{Limitações}

Esse breve relatório está limitado a achados relacionados a FC e o uso de metoprolol, e não tem um grupo de comparação. Os autores concordam que um desenho prospectivo teria sido robusto. Entretanto, essa foi uma revisão retrospectiva que analisou nossa prática clínica. Também é importante mencionar que os leitores deste estudo não fizeram a leitura cega, o que poderia introduzir um viés.

\section{Conclusão}

Um protocolo de dose de metoprolol na população pediátrica externa com DCC, antes da aquisição da CCTA, demonstrou ser segura e eficaz na redução de frequência cardíaca em pacientes entre 6 e 18 anos de idade. Um controle de frequência cardíaca adequado na população, utilizando o metoprolol, pode oferecer imagens mais claras devido à redução da movimentação e artefato, e garantindo tempo de aquisição menores e, portanto, associados à redução da exposição à radiação.

\section{Contribuição dos Autores}

Concepção e desenho da pesquisa: Casey SA, Chu BJ, Lesser JR, Han BK; Obtenção de dados: Casey SA, Caye DJ, Chu BJ, Lindberg BJ, Lesser JR, Han BK; Análise e interpretação dos dados: Casey SA, Chu BJ, Han BK; Análise estatística: Casey SA, Stanberry LI, Han BK; Obtenção de financiamento: Han BK; Redação do manuscrito e Revisão crítica do manuscrito quanto ao conteúdo intelectual importante: Nunes MO, Witt DR, Casey SA, Chu BJ, Han BK.

\section{Potencial Conflito de Interesses}

Declaro não haver conflito de interesses pertinentes.

\section{Fontes de Financiamento}

O presente estudo foi parcialmente financiado pela Jon Dehaan Foundation, Siemens Medical Solutions.

\section{Vinculação Acadêmica}

Não há vinculação deste estudo a programas de pósgraduação.

\section{Aprovação Ética e Consentimento Informado}

Este estudo foi aprovado pelo Comitê de Ética do Alina Health IRB sob o número de protocolo 1036442-1. Todos os procedimentos envolvidos nesse estudo estão de acordo com a Declaração de Helsinki de 1975, atualizada em 2013.

\section{Referências}

1. Budoff MJ, Achenbach S, Blumenthal RS, Carr JJ, Goldin JG, Greeland P, et al. Assessment of coronary artery disease by cardiac computed tomography: a scientific statement from the American Heart Association Committee on Cardiovascular Imaging and Intervention, Council on Cardiovascular Radiology and Intervention, and Committee on Cardiac Imaging, Council on Clinical Cardiology. Circulation. 2006;114(16):1761-91.

2. Cury RC, Abbara S, Achenbach S, Agatston A, Berman DS, Budoff MJ, et al. CAD-RADS(TM) Coronary Artery Disease - Reporting and Data System. An expert consensus document of the Society of Cardiovascular Computed Tomography (SCCT), the American College of Radiology (ACR) and the North American Society for Cardiovascular Imaging (NASCI). Endorsed by the American College of Cardiology. J Cardiovasc Comput Tomogr. 2016;10(4):269-81.

3. Han BK, Rigsby CK, Leipsic J, Bardo D, Abbara S, Ghoshhajra B, et al. Computed Tomography Imaging in Patients with Congenital Heart Disease, Part 2: Technical Recommendations. An Expert Consensus Document of the Society of Cardiovascular Computed Tomography (SCCT): Endorsed by the Society of Pediatric Radiology (SPR) and the North American Society of Cardiac Imaging (NASCI). J Cardiovasc Comput Tomogr. 2015; 9(6):493-513.

4. Achenbach S, Manolopoulos M, SchuhbäckA, Ropers D, Rixe J, Schneider C, et al. Influence of heart rate and phase of the cardiac cycle on the occurrence of motion artifact in dual-source CT angiography of the coronary arteries. J Cardiovasc Comput Tomogr. 2012;6(2):91-8.

5. Goo HW. Coronary artery imaging in children. Korean J Radiol. 2015;16(2):239-50.

6. Han BK, Lindberg J, Overman D, Schwartz RS, Grant K, Lesser JR. Safety and accuracy of dual-source coronary computed tomography angiography in the pediatric population. J Cardiovasc Comput Tomogr. 2012;6(4):252-9.

7. Cheng Z, Wang X, Duan Y, Wu L, Wu D, Chao B, et al. Low-dose prospective ECG-triggering dual-source CT angiography in infants and children with complex congenital heart disease: first experience. Eur Radiol. 2010;20(10):2503-11.

8. Frommelt P, Lopez L, Dimas VV, Srivastava S, Valente AM, Cohen MS, et al. Recommendations for Multimodality Assessment of Congenital Coronary Anomalies: A Guide from the American Society of Echocardiography: Developed in Collaboration with the Society for Cardiovascular Angiography and Interventions, Japanese Society of Echocardiography, and Society for CardiovascularMagnetic Resonance.J Am Soc Echocardiogr. 2020;33(3):259-94.

9. Han BK, Vezmar M, Lesser JR, Michalak G, Grant K, Dassenko D, et al. Selective use of cardiac computed tomography angiography: an alternative diagnostic modality before second-stage single ventricle palliation. J Thorac Cardiovasc Surg. 2014;148(4):1548-54.

10. Mahabadi AA, Achenbach S, Burgstahler C, Dill T, Fischbach R, Knez A, et al. Safety, efficacy, and indications of-adrenergic receptor blockade to reduce heart rate prior to coronary CT angiography. Radiology. 2010;257(3):614-23. 
11. Sabarudin A, Sun Z. Beta-blocker administration protocol for prospectively ECG-triggered coronary CT angiography. World J Cardiol. 2013;5(12):453-8.

12. Li M, Zhang G-M, Zhao J-S, Jiang ZW, Peng ZH, Jin ZT, et al. Diagnostic performance of dual-source CT coronary angiography with and without heart rate control: systematic review and meta-analysis. Clin radiol. 2014;69(2):163-71.

13. Watanabe $H$, Kamiyama $H$, Kato $M$, Komori A, Abe $\mathrm{Y}$, Ayusawa $M$. Appropriate use of a beta-blocker in paediatric coronary $\mathrm{CT}$ angiography. Cardiol Young. 2018;28(10):1148-53.

14. Team R. RStudio: integrated development for R. Boston, MA: RStudio. In: Inc; 2015

15. Team RC. R: a language and environment for statistical computing. Version 3.1. 1 [computer program]. R Foundation for Statistical Computing, Vienna, Austria. In:2014.

16. de Graaf FR, Schuijf JD, van Velzen JE, Kroft LJ, Roos A, Sieders A, et al. Evaluation of contraindications and efficacy of oral Beta blockade before computed tomographic coronary angiography. Am J Cardiol. 2010;105(6):767-72
17. Flick RP, Katusic SK, Colligan RC, Wilder RT, Voigt RG, Olson MD, etal. Cognitive and behavioral outcomes after early exposure to anesthesia and surgery. Pediatrics. 2011;128(5):e1053-61

18. Ing C, DiMaggio C, Whitehouse A, Hegarty MK, Brady J, von Ungern-Sternberg $\mathrm{BS}$, etal. Long-term differences in language and cognitive function after childhood exposure to anesthesia. Pediatrics. 2012;130(3):e476-85.

19. Mathews JD, Forsythe AV, Brady Z, Butler MW, Goergen SK, Byrnes GB, et al. Cancer risk in 680,000 people exposed to computed tomography scans in childhood or adolescence: data linkage study of 11 million Australians. BMJ. 2013;346:f2360.

20. Ramamoorthy C, Haberkern CM, Bhananker SM, Domino KB, Posner KL, Campos $\mathrm{IS}$, et al. Anesthesia-related cardiac arrest in children with heart disease: data from the Pediatric Perioperative Cardiac Arrest (POCA) registry. Anesth Analg. $2010 \cdot 110(5): 1376-82$

21. Gottlieb EA, Andropoulos DB. Anesthesia for the patient with congenita heart disease presenting for noncardiac surgery. Curr Opin Anaesthesiol. 2013;26(3):318-26.

22. Menke J, Unterberg-Buchwald C, Staab W, Sohns JM, Seif Amir Hosseini A SchwarzA. Head-to-head comparison of prospectively triggered vs retrospectively gated coronary computed tomography angiography: Meta-analysis of diagnostic accuracy, image quality, and radiation dose. Am HeartJ. 2013;165(2):154-63.e3. 